\title{
Smac peptide potentiates TRAIL- or paclitaxel-mediated ovarian cancer cell death in vitro and in vivo
}

\author{
HONG LUAN MAO, YINGXIN PANG, XIAOLEI ZHANG, FANG YANG, \\ JINGFANG ZHENG, YU WANG and PEISHU LIU
}

Department of Obstetrics and Gynecology, Qilu Hospital of Shandong University, Jinan, Shandong 250012, P.R. China

Received August 6, 2012; Accepted September 28, 2012

DOI: $10.3892 /$ or.2012.2132

\begin{abstract}
Second mitochondria-derived activator of caspases (Smac) is a recently identified protein that is released from mitochondria in response to apoptotic stimuli and promotes apoptosis by antagonizing the inhibitor of apoptosis proteins (IAPs). Our previous study showed that ectopic overexpression of Smac sensitizes drug-resistant tumor cells to TRAIL- or paclitaxel-induced apoptosis in vitro. The present study was designed to explore the effect of the synthesized Smac N7 peptide in a human ovarian cancer cell line and xenograft model. The results showed that the single-agent Smac N7 had a non-cytotoxic effect, but it effectively enhanced TRAIL- or paclitaxel-induced inhibition of cell proliferation in a dosedependent manner, even in TRAIL-resistant A2780 cells. When Smac N7 was combined with TRAIL or paclitaxel in treating A2780 cell tumor xenografts, synergistic anticancer effects were achieved. Furthermore, the combination therapy caused less damage in normal tissues and more apoptosis in tumor xenografts compared with TRAIL or paclitaxel alone. Increased apoptosis was associated with the downregulation of XIAP, survivin and the increased activity of caspase-3, along with an increased amount of cleaved PARP. In conclusion, this Smac N7 peptide is a promising candidate for ovarian cancer combination therapy, and Smac may be the target for the development of a novel class of anticancer drugs.
\end{abstract}

\section{Introduction}

Abnormalities in the control of the execution of apoptosis, which contributes to enhancement of tumor progression and treatment resistance, are observed in many malignancies, including ovarian cancer (1). Tumor cells often circumvent the apoptotic cascade and proliferate in the face of apoptotic

Correspondence to: Dr Peishu Liu, Department of Obstetrics and Gynecology, Qilu Hospital, Shandong University, No. 107 Wen Hua Xi Road, Jinan, Shandong 250012, P.R. China

E-mail: peishuliu@126.com

Key words: second mitochondria-derived activator of caspase, peptide, apoptosis, epithelial ovarian cancer, TRAIL, paclitaxel stimuli, which facilitates tumor progression and metastasis (2). Thus, targeting key apoptosis regulators with a goal to overcome apoptosis resistance of tumor cells has become the focus of extensive pharmaceutical research.

Two main pathways leading to apoptosis-associated caspase activation have been identified: the extrinsic and the intrinsic apoptotic pathway (3). In the extrinsic death receptor pathway, apoptosis is initiated through the binding of death receptor superfamily proteins, such as TRAIL, to their respective cell surface receptors, leading to caspase- 8 activation. In the intrinsic mitochondrial pathway, a variety of apoptotic stimuli, including anticancer agents, triggers changes in mitochondrial membrane permeability and the release of pro-apoptotic proteins including cytochrome $c$ and Smac. Cytochrome $c$ triggers caspase-3 activation through the apoptosome complex, whereas Smac promotes caspase-3 activation by binding to and neutralizing the inhibitor of the apoptotic proteins (IAPs) (4).

The IAPs are a family of key apoptosis regulators, characterized by one or more baculovirus IAP repeats (BIR) (5). Among them, X-linked IAP (XIAP), cellular IAP1 (cIAP1), cIAP2 and survivin, function to block death receptor and mitochondrial-mediated apoptotic pathways mainly by preventing the activation of procaspases and inhibiting the enzymatic activity of mature caspases. Evidence indicates that XIAP, survivin, cIAP1 and cIAP2 are overexpressed in human tumor tissues and their downregulation confers an apoptosis enhancement effect in chemoresistant human cancer cells (6). Smac interacts with IAPs, such as XIAP, CIAP-1, and CIAP-2, at the level of the BIR domains via its N-terminal AVPI terapeptide binding motif, thereby eliminating the inhibitory effects of IAPs on caspase-3, -7 and -9 (7-9). Smac peptides as short as 4-residue (AVPI), derived from the Smac protein bind to the recombinant XIAP BIR3 domain protein with the same affinities as the mature Smac protein (9). Previous studies have demonstrated that the first 4-8 N-terminal amino acids of Smac fused to the Drosophila antennapedia penetration sequence, a carrier peptide, were shown to enhance the induction of apoptosis of diverse antineoplastic agents including paclitaxel, etoposide, 7-ethyl-10-hydroxycamptothecin (SN-38), TRAIL and doxorubicin $(10,11)$.

Epithelial ovarian cancer (EOC) is the fifth most frequent cause of cancer-related death in women. The standard first-line therapy for ovarian cancer includes tumor debulking followed 
by chemotherapy treatment with paclitaxel, platinum-based agents, or combinations of both (12-14). Despite the initial response to treatment, the majority of patients relapse and eventually progress to a chemotherapy-resistant state (14). We previously demonstrated decreased Smac and increased XIAP expression in EOC tissues, and ectopic expression of Smac sensitized drug-resistant EOC cells to undergo apoptosis by TRAIL or paclitaxel (15). In the present study, we synthesized a cell permeable Smac peptide and evaluated its effect on the in vitro and in vivo therapeutic efficacy of various chemotherapy agents, in an effort to provide a more effective antitumor strategy for EOC.

\section{Materials and methods}

Cell line and cell culture. The human ovarian epithelial cancer cell line A2780 was provided by Qilu Hospital Biotechnology Center of Shandong University. Cells were cultured in RPMI-1640 medium (Gibco/BRL, Grand Island, NY, USA) supplemented with $10 \%$ heat-inactivated fetal bovine serum (FBS; Gibco/BRL) and incubated in an atmosphere of $5 \% \mathrm{CO}_{2}$ at $37^{\circ} \mathrm{C}$.

Design, synthesis and detection of peptides. The Smac N7 peptide and the control peptide (R-Smac N7) were designed by conjugating the $\mathrm{N}$-terminal seven amino acids of Smac, AVPIAQK, which are critical for its binding to IAPs or the random arrangement sequence of Smac, KIPAQVA with the Drosophila antennapaedia penetration sequence and fluorescein isothiocyanate (FITC). All of the peptides were custom-synthesized by Sangon Biotechnology (Shanghai). The purity of the peptides as determined by high-performance liquid chromatography was $>95 \%$.

Cell viability assay. Cell viability rate was quantitated using a modified methylthiazol tetrazolium (MTT) colorimetric assay. Cells were seeded into 96-well culture plates (4000/well), allowed to adhere overnight, and then treated with different concentrations of TRAIL (Chemicon, USA) or paclitaxel (Bristol-Myers Squibb Co., USA) in the absence or presence of diverse doses of Smac N7 or R-Smac N7 for $24 \mathrm{~h}$, respectively. At the end of each treatment, cells were incubated with $5 \mathrm{mg} /$ ml MTT (Sigma, USA) for $4 \mathrm{~h}$ and then mixed with dimethyl sulfoxide after the supernatant was removed. Cell viability was quantitated by reading the dye absorption (A) at $550 \mathrm{~nm}$ (A1) and $630 \mathrm{~nm}$ (A2) with an automatic multiwell spectrophotometer (Coda; Bio-Rad, Richmond, CA, USA). All the experiments were performed in triplicate and reproduced at least three times.

Flow cytometric analysis. Flow cytometric analysis was performed to identify the intracellular uptake rate of FITCtagged Smac N7 peptide and R-Smac N7 peptide. Briefly, A2780 cells $\left(5 \times 10^{6}\right.$ cells $\left./ \mathrm{ml}\right)$ were planted in culture flasks. After most of the cells became adherent, the cells were treated with $10 \mu \mathrm{mol} / 1 \mathrm{Smac}$ N7 or R-Smac N7 for $24 \mathrm{~h}$, respectively. All cells were collected and washed twice with phosphatebuffered saline after centrifugation, and intracellular uptake rates were determined by a flow cytometry assay (FACScan, Becton Dickinson, USA).
A

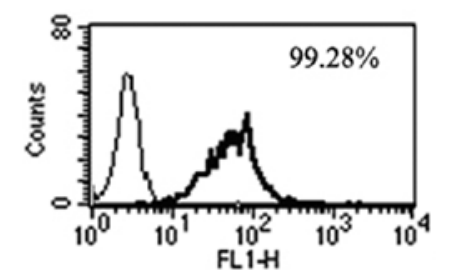

B

Smac peptide $10 \mu \mathrm{mol} / 1$

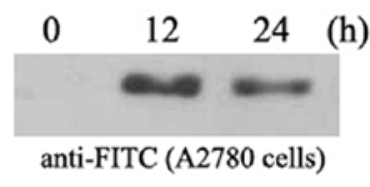

Figure 1. Cellular uptake of the synthetic Smac N7 peptide. (A) The uptake rate of Smac N7 peptide into the cytoplasm was analyzed by flow cytometry $24 \mathrm{~h}$ after the addition of a $10 \mu \mathrm{mol} / 1$ concentration. (B) Cells were treated with Smac N7 $(10 \mu \mathrm{mol} / \mathrm{l})$ for 12 and $24 \mathrm{~h}$. Anti-FITC antibodies were added to cell extracts to detect the cellular uptake of Smac N7 by western blotting.

Animal tumor models and treatment. Female nude mice (BALB/c, 4-5 weeks old) were purchased from the Shanghai Experimental Animal Center (Chinese Academy of Sciences, Shanghai). The mice were housed and maintained under specific pathogen-free conditions according to the experimental animal guidelines. A2780 cells were harvested and re-suspended in RPMI-1640 media. A2780 cells $(200 \mu \mathrm{l})\left(2 \times 10^{7} / \mathrm{ml}\right)$ were injected into the right flank of the mice. Two weeks later, the mice were randomly assigned to six groups with 5 mice in each group. A total of $0.1 \mathrm{ml}$ PBS, Smac N7 $(12.5 \mathrm{mg} / \mathrm{kg})$, TRAIL $(10 \mu \mathrm{g} / \mathrm{kg})$, paclitaxel $(20 \mathrm{mg} / \mathrm{kg})$, Smac N7 $(12.5$ $\mathrm{mg} / \mathrm{kg})+$ TRAIL $(10 \mu \mathrm{g} / \mathrm{kg})$, Smac N7 $(12.5 \mathrm{mg} / \mathrm{kg})+$ paclitaxel $(20 \mathrm{mg} / \mathrm{kg})$ were intratumorally injected seven times at an interval of 3 days, respectively. Tumor sizes were measured by length (l) and width (w) every 4 days, and the tumor volumes were calculated according to the following formula: Tumor volume $=1 \mathrm{x} \mathrm{w}^{2} / 2$. The mice were observed daily for evaluation of their mental state, diet and stools. Twelve hours following the last injection, the mice were sacrificed, and the tumors, hearts, livers, kidneys and spleens were removed. Part of the tissues were fixed in formalin, embedded in paraffin and stained with hematoxylin and eosin (H\&E), while others were frozen immediately in liquid nitrogen at $-80^{\circ} \mathrm{C}$ for the western blot assay. All animal studies were approved and carried out in accordance with the guidelines of the Experimental ethics review committee of Shandong University.

Western blotting. Cells or xenograft tumor tissues were lysed in lysis buffer. The whole cell extracts (30 $\mu \mathrm{g} / \mathrm{lane})$ were equally loaded on 10-12\% SDS-PAGE gels and transferred to nitrocellulose membranes (Millipore, Bedford, MA, USA) using the Bio-Rad electrotransfer system. The membrane was blocked in Tris-buffered saline with 5\% (w/v) nonfat dry milk, and then incubated with a primary antibody against XIAP, survivin, PARP (Cell Signaling Technology, Danvers, MA, USA), or Bcl-2, caspase-3, GAPDH, anti-FITC (Santa Cruz Biotechnology, Inc., Santa Cruz, CA, USA) overnight at $4^{\circ} \mathrm{C}$, respectively. After being washed and incubated with horseradish peroxidase conjugated second antibody, the proteins were visualized using an enhanced chemiluminescence (ECL) detection system (Pierce, Rockford, IL, USA). The bands were examined using a densitometer analysis system Flurochem 9900-50 (Alpha Innotech, San Leandro, CA, USA). Band density was analyzed using Bandscan soft- 

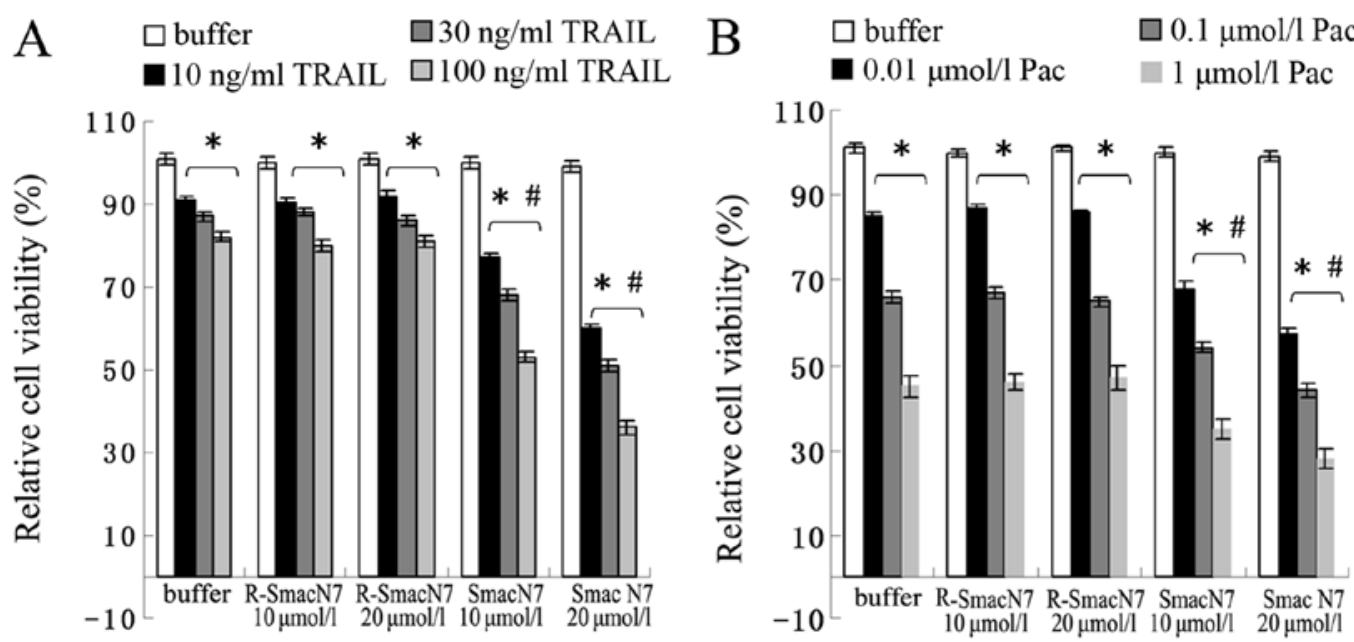

Figure 2. Smac N7 sensitized the TRAIL- or paclitaxel-induced growth inhibition of A2780 cells. A2780 cells were respectively co-treated with Smac N7 (10 or $20 \mu \mathrm{mol} / \mathrm{l})$, R-Smac N7 (10 or $20 \mu \mathrm{mol} / \mathrm{l})$, and/or buffer and different concentrations of TRAIL (10,30, $100 \mathrm{ng} / \mathrm{ml})$ (A) or paclitaxel (pac) (0.01, 0.1, $1 \mu \mathrm{mol} / \mathrm{l}$ ) (B), respectively for $24 \mathrm{~h}$. Cell viability was measured by MTT assay. "P $\mathrm{P} 0.05$, significantly different from the respective control; ; $\mathrm{P}<0.05$, significantly different from TRAIL or paclitaxel alone.

ware (Glyko, USA) and the results were expressed as a ratio of the protein of interest/GAPDH to correct for loading for each sample.

TUNEL detection of apoptotic tumor cells. TUNEL was performed with a Fluorometric TUNEL system (KeyGeen Biotech, Nanjing), following the manufacturer's protocol. Dissected tumors were rinsed with PBS for 5 min after incubation with proteinase $\mathrm{K}(18 \mu \mathrm{g} / \mathrm{ml})$ for $20 \mathrm{~min}$, and then blocked with fetal bovine serum for $15 \mathrm{~min}$ at room temperature. TdT reaction mix $(50 \mu \mathrm{l})$ was added to the section and incubated in a humidified chamber for $1 \mathrm{~h}$ at $37^{\circ} \mathrm{C}$. Then the sections were rinsed with PBS and assessed using a fluorescence microscope. Cell nuclei with green fluorescent staining were defined as TUNEL-positive nuclei. Cell apoptosis was quantitated by counting the number of green fluorescence-positive cells in five random fields of view in each section at a magnification of $\mathrm{x} 200$.

Data analysis and statistics. The values are presented as means $\pm \mathrm{SD}$, and were statistically evaluated by analysis of variance (ANOVA). Statistical analysis was performed with SPSS software (version 13.0; SPSS Inc., Chicago, IL). $\mathrm{P}<0.05$ was considered to indicate a statistically significant result in all cases.

\section{Results}

Cellular uptake of the Smac N7 peptide. Following exposure to $10 \mu \mathrm{mol} / 1$ of Smac N7 peptide for $24 \mathrm{~h}$, the intracellular uptake of FITC-tagged Smac N7 in A2780 cells was 99.28\% (Fig. 1A), as confirmed by flow cytometric analysis (FCM). We then assessed the cellular uptake of Smac N7 using western blotting. After A2780 cells were treated with Smac N7 (10 $\mu \mathrm{mol} / \mathrm{l})$ for 12 or $24 \mathrm{~h}$, cells were harvested and subjected to western blotting with anti-FITC antibodies. The synthetic Smac N7 peptide was successfully taken up into the cytoplasm of A2780 cells, and the uptake was most prominent after $12 \mathrm{~h}$ (Fig. 1B).

Smac N7 peptide sensitizes A2780 cells to TRAIL-or paclitaxel-induced cell growth inhibition. Our previous study showed that ectopic expression of the Smac gene enhanced the therapeutic potential of TRAIL or paclitaxel in ovarian cancer cells (15). In this study, we explored whether the Smac N7 peptide has a similar effect. As shown in Fig. 2, the results indicated that the administration of TRAIL or paclitaxel, but not PBS or Smac N7, resulted in cell growth inhibition in A2780 cells in a dose-dependent manner. Moreover, compared with cells treated with TRAIL or paclitaxel alone, significantly reduced cell viability was observed in cells treated with TRAIL or paclitaxel accompanied with Smac N7, respectively, and Smac N7 had a dose-dependent effect on TRAIL or paclitaxel sensitization in A2780 cells.

Co-treatment with the Smac N7 peptide potentiates TRAILor paclitaxel-induced tumor regression effect and apoptosis in A2780 cell xenografts in nude mice. Given the TRAIL- or paclitaxel-sensitizing properties of Smac N7 in vitro, we corroborated these findings in BALB/c mice bearing A2780 cell xenografts. A2780 cells were implanted into the right flank of nude mice. The rate of tumor formation was $100 \%$, and the average time was $\sim 12-14$ days. On day 38 after implantation, the A2780 cell tumors of mice treated with PBS and Smac N7 reached $2021.74 \pm 123.19$ and $1893.00 \pm 131.27 \mathrm{~mm}^{3}$ in volume, respectively $(\mathrm{P}>0.05)$. The volumes of tumors treated with TRAIL, paclitaxel, Smac N7+TRAIL or Smac N7+paclitaxel were $1476.50 \pm 188.90,1188.25 \pm 141.24,604.40 \pm 50.04$ and $522.50 \pm 75.48 \mathrm{~mm}^{3}$, respectively, which were significantly smaller than those treated with PBS or Smac N7 alone $(\mathrm{P}<0.05)$ (Figs. 3 and 4). Noticeably, the combination of Smac N7 and TRAIL or paclitaxel resulted in a significant reduction in tumor volume compared with the treatment of each drug alone $(\mathrm{P}<0.05)$. 


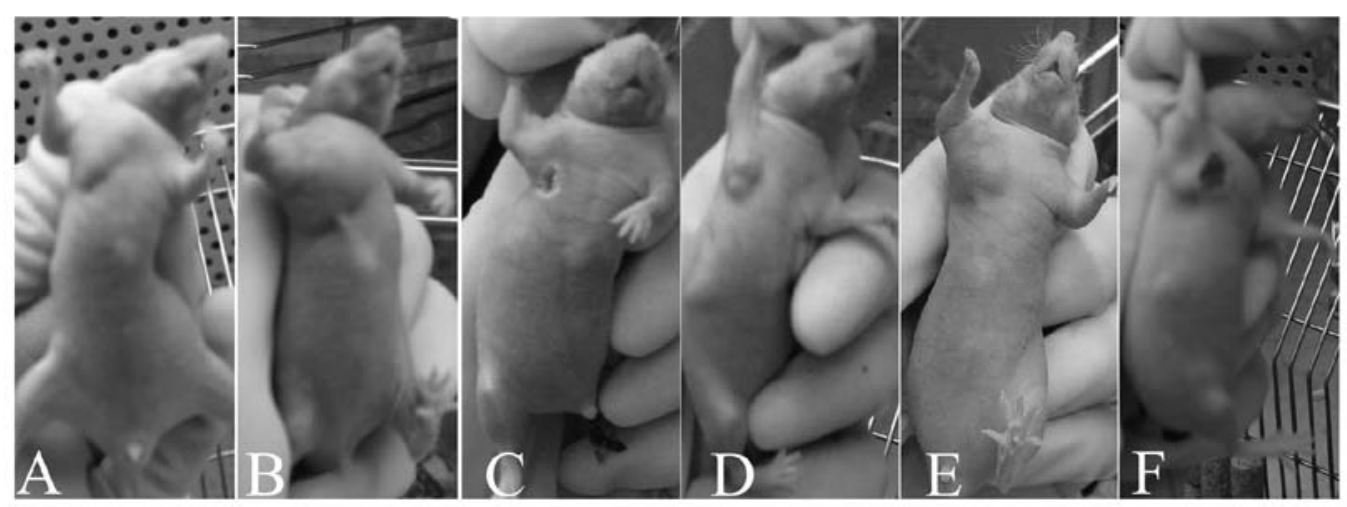

Figure 3. EOC tumor models in nude mice. (A) PBS group; (B) Smac N7 peptide group; (C) Smac N7 peptide+paclitaxel group; (D) Smac N7 peptide+TRAIL group; (E) TRAIL group; (F) paclitaxel group.

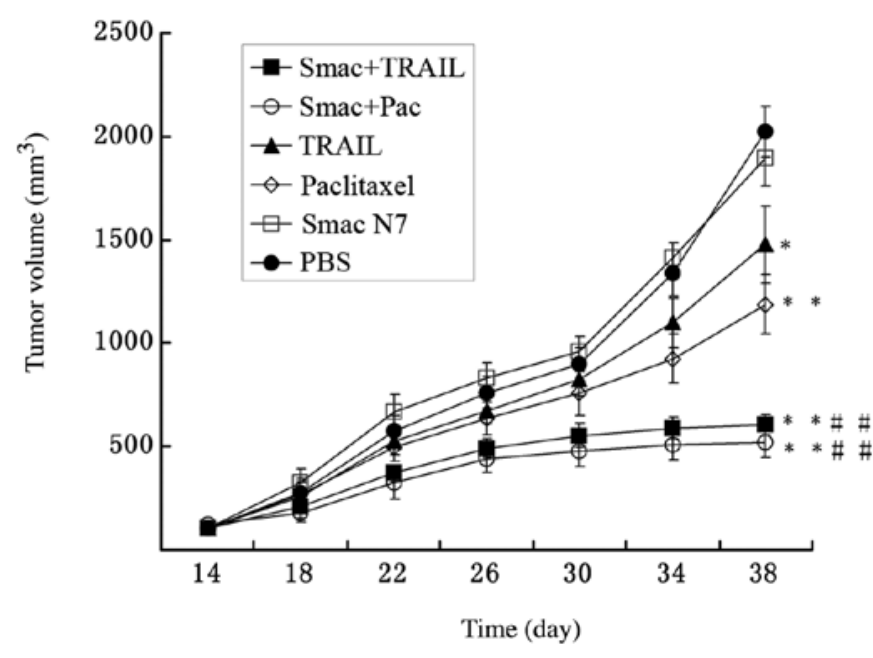

Figure 4. Tumor growth curves showed that the Smac N7 peptide in combination with TRAIL or paclitaxel (pac) was effective in controlling EOC xenografts. Each point represented the average of the volume of all tumor xenografts $\pm \mathrm{SE} .{ }^{*} \mathrm{P}<0.01$, significantly different from the control groups (Smac N7 peptide and PBS group); ${ }^{* *} \mathrm{P}<0.001$, significantly different from the control groups (Smac N7 peptide and PBS group); ${ }^{\#} \mathrm{P}<0.001$, significantly different from TRAIL or paclitaxel alone group.

Fluorometric TUNEL assay was performed to detect the apoptotic cells in the tumor tissues of nude mice. As shown in Fig. 5, the apoptotic cells accounted for $41 \pm 7.65$ or $53 \pm 6.29$ in the TRAIL or paclitaxel-treated group versus 12.8 \pm 3.11 in the Smac N7-treated group and 10.6 \pm 3.0 in PBS control group $(\mathrm{P}<0.05)$, and the apoptotic cells accounted for $79.2 \pm 12.19$ or $96 \pm 12.39$ in the combination treatment groups (Smac N7+TRAIL or Smac N7+paclitaxel, respectively). These results suggest that Smac N7 alone had no significant apoptosis-inducing effect on A2780 cell tumor xenografts, but further enhanced the apoptosis induction by TRAIL or paclitaxel.

Smac N7 peptide reduces the cytotoxic effect of TRAIL or paclitaxel on vital organs of nude mice. Although paclitaxel has demonstrated clinical efficacy in most types of human cancers, adverse vital organ pathologies and toxicities continue to be of major concern. In our study, necrosis of mice skin around the injection point was observed in both the paclitaxel and Smac N7+paclitaxel treatment groups, but the range of skin necrosis seemed smaller in the latter group (Fig. 3). We also carried out H\&E staining of the transplanted tumors and the heart, liver, kidney and spleen of the nude mice. The karyomegaly, anachromasis, and karyokinesis appearance observed in tumor sections confirmed the formation of xenograft tumors. No obvious damage was found in the examined organs of the mice treated with PBS or Smac N7. Compared with TRAIL, paclitaxel showed severe cytotoxicity to the liver and kidney of mice. In particular, the damage to the mice treated with the combination therapy of Smac N7 and paclitaxel or TRAIL was markedly less than that experienced by the mice treated with paclitaxel or TRAIL alone. Notably, apart from the PBS-treated mice, the spleen of all tested mice displayed an enlarged volume with blood stasis and infiltrating mononuclear phagocytes (Table I).

Smac N7 peptide increases TRAIL-or paclitaxel-induced caspase-3 and PARP activation and downregulates the protein levels of the IAP family. To gain insight into the mechanisms by which the combination of Smac N7 peptide and TRAIL or paclitaxel exerts enhanced anticancer activity, we analyzed the effects of the combination therapy on caspase-3 activity and on the expression of XIAP, survivin and Bcl-2 in comparison with either agent alone. Evaluation of cell lysates revealed that the Smac N7 peptide had no effect on caspase activation and only a slightly downregulatory effect on XIAP protein expression, but potentiated TRAIL- or paclitaxel-induced caspase-3 activation as represented by increased levels of cleaved caspase- 3 and PARP, as well as enhanced TRAIL- or paclitaxel-induced downregulation of XIAP and survivin in the xenograft tumor tissues (Fig. 6). Notably, paclitaxel resulted in the downregulation of Bcl-2, but paclitaxel plus the Smac N7 peptide did not have an additive effect. Furthermore, no modification of Bcl-2 was observed in the TRAIL-treated tumor model.

\section{Discussion}

Our previous study showed that the disrupted balance of XIAP and Smac expression in EOC tissues, and ectopic Smac expression reversed the resistance to paclitaxel and TRAIL of EOC cell lines (15). According to these observations, it 
Table I. Pathologic examination of nude mice after treatment: the H\&E staining of the heart, liver, kidney and spleen.

\begin{tabular}{|c|c|c|c|c|}
\hline \multirow[b]{2}{*}{ Group } & \multicolumn{4}{|c|}{ Organ } \\
\hline & Heart & Liver & Spleen & Kidney \\
\hline PBS & Normal & Normal & Normal & Normal \\
\hline Smac N7 & Normal & Normal & $\begin{array}{l}\text { Volume enlarged, blood } \\
\text { stasis, a large number of } \\
\text { infiltrating mononuclear } \\
\text { phagocytes were } \\
\text { observed (no. } 1-5 \text { ) }\end{array}$ & Normal \\
\hline TRAIL & $\begin{array}{l}\text { No obvious change } \\
\text { in myocardial cells }\end{array}$ & $\begin{array}{l}\text { Normal } \\
\text { (no. } 1-3 \text { ) } \\
\text { ballooning } \\
\text { degeneration } \\
(\text { no. } 4,5)\end{array}$ & $\begin{array}{l}\text { Volume enlarged, blood } \\
\text { stasis, a large number of } \\
\text { infiltrating mononuclear } \\
\text { phagocytes were } \\
\text { observed (no. } 1-5 \text { ) }\end{array}$ & Normal \\
\hline Paclitaxel & $\begin{array}{l}\text { No obvious change } \\
\text { in myocardial cells } \\
\text { (no. 1-5), } \\
\text { vasodilatation with } \\
\text { excessive } \\
\text { accumulation of } \\
\text { neutrophilic } \\
\text { granulocytes in } \\
\text { vessel lumen } \\
\text { among cardiac } \\
\text { muscle was } \\
\text { distinct (no. 2-4) }\end{array}$ & $\begin{array}{l}\text { Significant } \\
\text { hepatocellular } \\
\text { degeneration, } \\
\text { necrosis in } \\
\text { local liver } \\
\text { cells, small } \\
\text { abscess, central } \\
\text { vein dilation in } \\
\text { central portal tract } \\
\text { area (no. 1-5) }\end{array}$ & $\begin{array}{l}\text { Volume enlarged, blood } \\
\text { stasis, a large number of } \\
\text { infiltrating mononuclear } \\
\text { phagocytes was } \\
\text { observed (no. } 1-5) \text {, small } \\
\text { abscess (no. } 3,4)\end{array}$ & $\begin{array}{l}\text { Glomerular } \\
\text { capillary plexus } \\
\text { volume enlarged, } \\
\text { glomerular cyst } \\
\text { cavity became } \\
\text { narrow, leukocytes } \\
\text { accumulated in } \\
\text { capillary plexus. } \\
\text { No cast in } \\
\text { kidney tubules, } \\
\text { few neutrophilic } \\
\text { granulocytes in } \\
\text { renal interstitium } \\
\text { (no. 1-5) }\end{array}$ \\
\hline $\begin{array}{l}\text { Smac N7 } \\
+ \text { TRAIL }\end{array}$ & $\begin{array}{l}\text { No obvious change } \\
\text { in myocardial cells }\end{array}$ & Normal & $\begin{array}{l}\text { Volume enlarged, blood } \\
\text { stasis, a large number of } \\
\text { infiltrating mononuclear } \\
\text { phagocytes were } \\
\text { observed (no. } 1-5 \text { ) }\end{array}$ & Normal \\
\hline $\begin{array}{l}\text { Smac } \\
\mathrm{N} 7+\mathrm{Pac}\end{array}$ & $\begin{array}{l}\text { No obvious change } \\
\text { in myocardial cells }\end{array}$ & $\begin{array}{l}\text { Hepatocellular } \\
\text { degeneration, } \\
\text { nidus for abscesses } \\
\text { appeared in } \\
\text { individual areas }\end{array}$ & $\begin{array}{l}\text { Volume enlarged, blood } \\
\text { stasis, a large number of } \\
\text { infiltrating mononuclear } \\
\text { phagocytes were } \\
\text { observed (no. } 1-5)\end{array}$ & $\begin{array}{l}\text { Almost normal, } \\
\text { individual kidney } \\
\text { glomerulus had a } \\
\text { similar appearance } \\
\text { as the paclitaxel } \\
\text { treatment group }\end{array}$ \\
\hline
\end{tabular}

No. 1, 2, 3, 4, 5; the mouse number in each group.

was proposed that overexpression of Smac might be a potent strategy for EOC treatment by sensitization of tumor cells to chemotherapeutic drugs, yet this procedure has not been developed at the clinical level.

Based on reports that the suppression of apoptosis by the IAPs is primarily mediated either directly via their baculoviral IAP repeat (BIR) domains or indirectly through their ubiquitin E3 ligase RING domain, and that Smac through its N-terminal AVPI tetrapeptide binding motif interacts with both BIR2 and BIR3 domains of multiple IAPs to release the binding of IAPs to both initiator (caspase-9) and effector (caspase-3/7) caspases (16-18), we synthesized a cell permeable Smac peptide containing the N-terminal 7 amino acids of Smac and a Drosophila antennaaedia penetration sequence. The Smac N7 peptide appeared to have excellent cellular uptake and the amount of cytoplasmic accumulated Smac N7 in most cells peaked at $12 \mathrm{~h}$ after addition, as demonstrated by FCM and western blot analysis after 12 and $24 \mathrm{~h}$ of exposure.

TRAIL, a member of the TNF family, has been proposed as a cancer-specific therapeutic agent, for its rapid apoptosisinducing effect and non-toxic effects on normal tissues when 

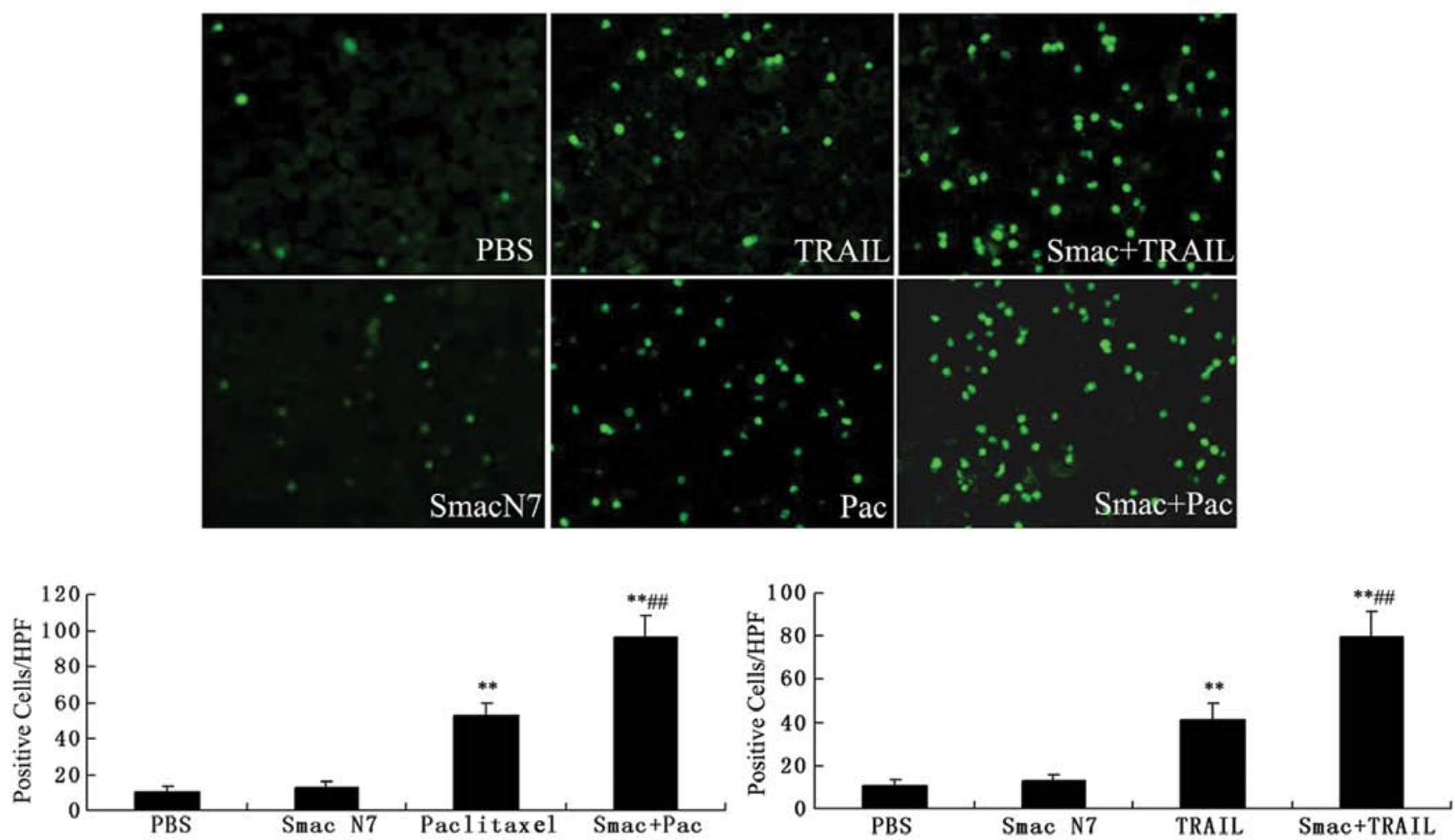

Figure 5. TUNEL assay indicates that Smac N7 peptide in combination with TRAIL or paclitaxel promotes ovarian cancer cell apoptosis in vivo. Cells with nuclei exhibiting green fluorescent staining were defined as apoptotic cells. The histogram showed that treatment with Smac N7 alone had no significant apoptosis-inducing effect, while TRAIL and paclitaxel induced cell apoptosis in EOC xenografts. The number of apoptotic cells in the combination treatment groups was increased when compared to the number in the single treatment groups. ${ }^{* *} \mathrm{P}<0.01$, significantly different from the PBS and Smac N7 peptide groups; ${ }^{\# \prime} \mathrm{P}<0.01$, significantly different from TRAIL or paclitaxel alone group.

systematically administered (19). Nevertheless, although TRAIL is well tolerated in patients, its single-agent efficacy is very limited and not all tumor cell lines respond well $(20,21)$. Paclitaxel has emerged as the first-line therapy for EOC or recurrent EOC, however, its clinical application is usually limited by the dose-dependent toxicity and development of drug resistance $(22,23)$. Therefore, combination chemotherapy is usually required to achieve efficacy at tolerable dosages. Herein, we demonstrated that even the A2780 cell line, which revealed resistance to TRAIL as shown by our previous study (15), could be strongly sensitized by the addition of Smac N7 to TRAIL treatment in a dose-dependent manner. Co-treatment with Smac N7 had the ability to potentiate the cytotoxicity of paclitaxel, which offers the potential to broaden the efficacy of previously established therapies in EOC.

However, we found that as a single agent, Smac N7 did not exhibit cytotoxicity. The non-cytotoxic effect induced by Smac N7 alone may be explained by two reasons. First, as demonstrated by western blot analysis, the expression levels of XIAP and survivin protein in A2780 cells are extremely high, which suggests that the Smac N7 peptide might not be sufficient to inhibit their antiapoptotic function. Second, recent studies have revealed that endogenous Smac protein is rapidly degraded after being released from mitochondria by the proteasome, and XIAP functions as an E3 ligase and promotes the degradation of Smac during apoptosis $(24,25)$. Thus, it could be considered that XIAP promotes the degradation of synthetic Smac N7 peptide in A2780 cells.
Based on the response observed in vitro, we corroborated these findings in vivo. Short Smac peptides fused to a carrier peptide for intracellular delivery were shown to overcome the resistance of cancer cells with high levels of IAPs and to enhance the activity of anticancer drugs in a human glioma xenograft model in vivo (26). Consistent with this study, our results revealed that in the A2780 xenograft models, intratumoral injection of the Smac N7 peptide combined with paclitaxel or TRAIL, caused an additional decrease in tumor load compared to each agent alone. The toxicity and side effects of antitumor drugs are always the focus of great concern to oncologists. In our study, the Smac N7 peptide exhibited no toxicity to vital organs, such as the heart, kidney and liver of nude mice; TRAIL showed very slight detectable damage, whereas the mice treated with paclitaxel showed poor general conditions, such as the decrease in immune function, and obvious damage to the kidney and liver. Notably, in the combination treatment groups, administration of Smac N7 did not reverse the lack of toxicity of TRAIL, and significantly reduced the toxicity of paclitaxel. In addition, except for the PBS-treated mice, the spleen of all tested mice displayed an enlarged volume with blood stasis and infiltrating mononuclear phagocytes. It could be hypothesized that the immune system is involved in the activity of the Smac N7 peptide, which was supported by a recently published report that a Smac mimetic elicited a proinflammatory cell death that was sufficient to activate adaptive antitumor immune responses in cancer (27). 

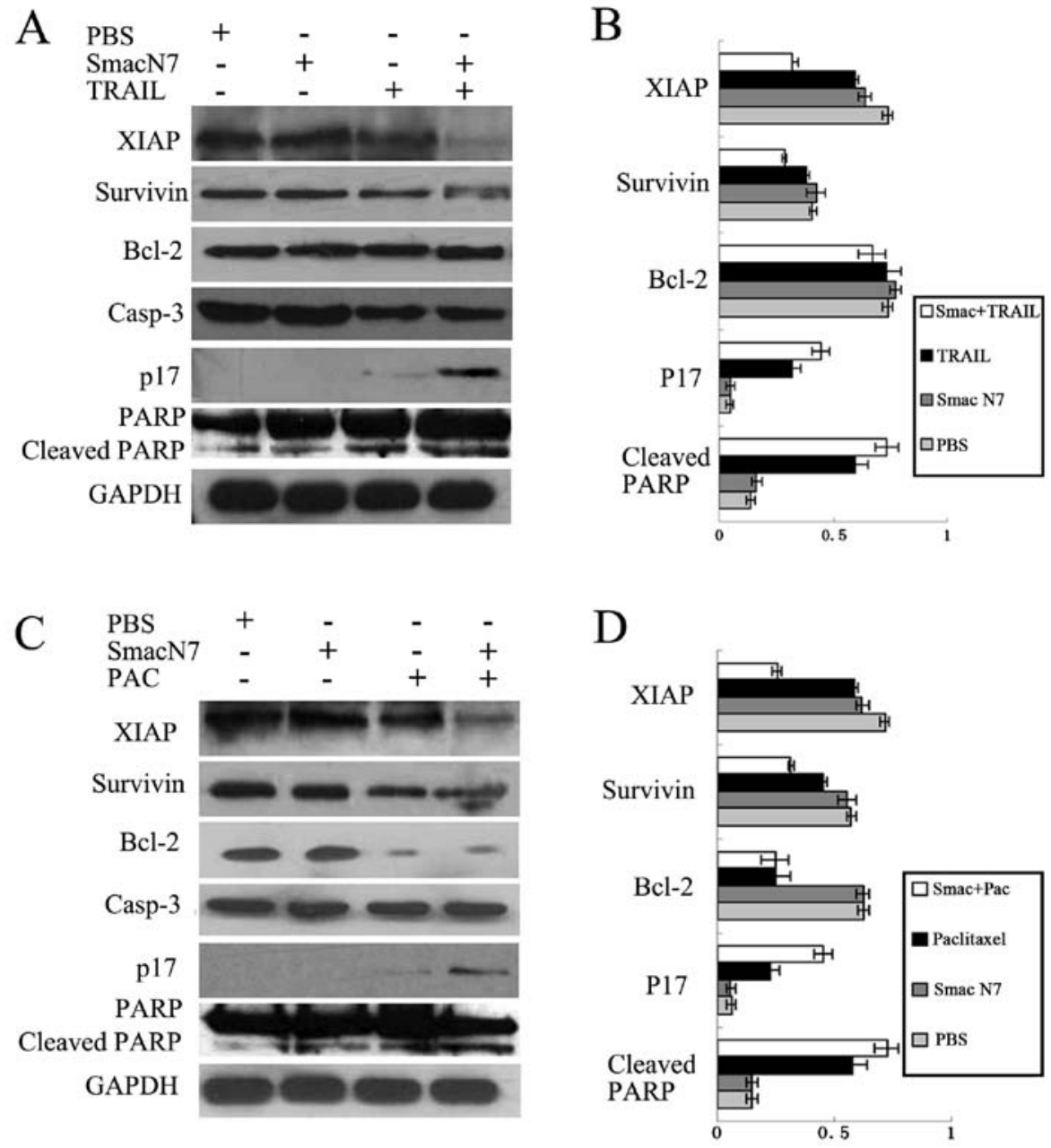

Figure 6. Smac N7 peptide potentiates TRAIL (A)- or paclitaxel (C)-induced tumor growth inhibition by promoting caspase-3 activation and degradation of XIAP and survivin in vivo. All protein lysates obtained from the xenografts were subjected to western blot analysis with antibodies against XIAP, survivin, Bcl-2, caspase-3 and PARP. GAPDH is shown as a loading control. As shown by band intensities which were determined by the OD value, combination treatment of A2780 cells with the Smac N7 peptide and TRAIL (B) or paclitaxel (D) enhanced the activation of caspase-3, as well as downregulated the protein level of XIAP and survivin. The bcl-2 protein levels in cells treated with TRAIL or TRAIL+Smac N7 exhibited no significant changes compared with the control and Smac N7 treatment group, while its levels in cells treated with paclitaxel or pac+Smac N7 were significantly decreased.

We next explored whether the growth inhibition of A2780 cell xenografts after combination therapy was in part due to induction of apoptosis. Detection of apoptotic cells by fluorometric TUNEL assay demonstrated that Smac N7 alone did not induce apoptosis, while paclitaxel or TRAIL moderately induced apoptosis while the combination of Smac N7 and paclitaxel or TRAIL showed additive effects. The accumulation of cleaved PARP and caspase-3 in the treated A2780 xenograft tumor cells further confirmed the induction of the apoptotic process.

Concerning the mechanism of action of the Smac N7 peptide, several recent studies showed that this peptide enhanced the apoptosis-inducing effect of cytotoxic drugs through the rapid degradation of XIAP and survivin $(28,29)$. In the present study, although the level of XIAP protein in A2780 tumor xenografts was slightly downregulated and the survivin protein level was not affected by Smac N7 alone, their expression levels were significantly downregulated following the combination treatment with paclitaxel or TRAIL. We hypothesized that this result was probably associated with the ability to reduce the protein level of XIAP by paclitaxel or
TRAIL, which relieved the E3 ligase effect of XIAP on the degradation of Smac protein. Additionally, it should be noted that $\mathrm{Bcl}-2$ expression remained unchanged in the Smac N7, TRAIL or the combination-treated A2780 tumor models. Paclitaxel reduced the expression of $\mathrm{Bcl}-2$, but the co-treatment with Smac N7 did not have an additive effect. Collectively, these data provide strong evidence that XIAP and survivin are the primary cellular targets for Smac N7 in its synergistic interaction with paclitaxel or TRAIL.

In summary, our data showed that the combination therapy of paclitaxel or TRAIL in conjugation with the Smac N7 peptide offers a promising strategy for the treatment of human epithelial ovarian cancer. Administration of Smac N7 did not reverse the lack of toxicity of TRAIL for normal tissues, and the combination treatments of Smac N7 and paclitaxel allowed a reduction in the therapeutically active dose of paclitaxel for EOC. These effects are, at least in part, mediated by the reduction in XIAP and survivin expression. Therefore, the combination of the Smac N7 peptide with TRAIL or paclitaxel presents a promising strategy for apoptosis-targeted therapies of EOC and warrants further investigation. 


\section{References}

1. Lee JY, Shin JY, Kim HS, et al: Effect of combined treatment with progesterone and tamoxifen on the growth and apoptosis of human ovarian cancer cells. Oncol Rep 27: 87-93, 2012.

2. Fesik SW: Promoting apoptosis as a strategy for cancer drug discovery. Nat Rev 5: 876-885, 2005.

3. Sayers TJ: Targeting the extrinsic apoptosis signaling pathway for cancer therapy. Cancer Immunol Immunother 60: 1173-1180, 2011.

4. Qiu W, Liu H, Sebastini A, Sun Q, Wang H, Zhang L and Yu J: An apoptosis-independent role of SMAC in tumor suppression. Oncogene: Jul 2, 2012 (Epub ahead of print).

5. Fulda S and Vucic D: Targeting IAP proteins for therapeutic intervention in cancer. Nat Rev Drug Discov 11: 109-124, 2012.

6. De Almagro MC and Vucic D: The inhibitor of apoptosis (IAP) proteins are critical regulators of signaling pathways and targets for anti-cancer therapy. Exp Oncol 34: 200-211, 2012.

7. Chai J, Du C, Wu JW, Kyin S, Wang X and Shi Y: Structural and biochemical basis of apoptotic activation by Smac/DIABLO. Nature 406: 855-862, 2000.

8 Du C, Fang M, Li Y, Li L and Wang X: Smac, a mitochondrial protein that promotes cytochrome c-dependent caspase activation by eliminating IAP inhibition. Cell 102: 33-42, 2000.

9. Liu Z, Sun C, Olejniczak ET, et al: Structural basis for binding of Smac/DIABLO to the XIAP BIR3 domain. Nature 408: 1004-1008, 2000.

10. Arnt CR, Chiorean MV, Heldebrant MP, Gores GJ and Kaufmann SH: Synthetic Smac/DIABLO peptides enhance the effects of chemotherapeutic agents by binding XIAP and cIAP1 in situ. J Biol Chem 277: 44236-44243, 2002.

11. Giagkousiklidis S, Vogler M, Westhoff MA, Kasperczyk H, Debatin KM and Fulda S: Sensitization for gamma-irradiationinduced apoptosis by second mitochondria-derived activator of caspase. Cancer Res 65: 10502-10513, 2005.

12. Rein DT, Volkmer AK, Volkmer J, et al: Systemic administration of bevacizumab prolongs survival in an in vivo model of platinum pre-treated ovarian cancer. Oncol Lett 3: 530-534, 2012.

13. Krasner CN, Poveda A, Herzog TJ, et al: Patient-reported outcomes in relapsed ovarian cancer: Results from a randomized Phase III study of trabectedin with pegylated liposomal doxorubicin (PLD) versus PLD Alone. Gynecol Oncol 127: 161-167 2012.

14. Mulier S, Claes JP, Dierieck V, et al: Survival benefit of adding Hyperthermic IntraPEritoneal Chemotherapy (HIPEC) at the different time-points of treatment of ovarian cancer: review of evidence. Curr Pharm Des 18: 3793-3803 2012.

15. Mao HL, Liu PS, Zheng JF, Zhang PH, Zhou LG, Xin G and Liu C: Transfection of Smac/DIABLO sensitizes drug-resistant tumor cells to TRAIL or paclitaxel-induced apoptosis in vitro. Pharmacol Res 56: 483-492, 2007.

16. Oost TK, Sun C, Armstrong RC, et al: Discovery of potent antagonists of the antiapoptotic protein XIAP for the treatment of cancer. J Med Chem 47: 4417-4426, 2004.
17. Bianchi A, Ugazzi M, Ferrante L, Lecis D, Scavullo C, Mastrangelo E and Seneci P: Rational design, synthesis and characterization of potent, drug-like monomeric Smac mimetics as pro-apoptotic anticancer agents. Bioorg Med Chem Lett 22: 2204-2208, 2012.

18. Yang D, Zhao Y, Li AY, Wang S, Wang G and Sun Y: Smacmimetic compound SM-164 induces radiosensitization in breast cancer cells through activation of caspases and induction of apoptosis. Breast Cancer Res Treat 133: 189-199, 2012.

19. Voelkel-Johnson C: TRAIL-mediated signaling in prostate, bladder and renal cancer. Nat Rev Urol 8: 417-427, 2011.

20. Kauntz H, Bousserouel S, Gossé F and Raul F: The flavonolignan silibinin potentiates TRAIL-induced apoptosis in human colon adenocarcinoma and in derived TRAIL-resistant metastatic cells. Apoptosis 17: 797-809, 2012.

21. Fandy TE, Shankar S and Srivastava RK: Smac/DIABLO enhances the therapeutic potential of chemotherapeutic drugs and irradiation, and sensitizes TRAIL-resistant breast cancer cells. Mol Cancer 7: 60, 2008.

22. Boere IA and van der Burg ME: Review of dose-intense platinum and/or paclitaxel containing chemotherapy in advanced and recurrent epithelial ovarian cancer. Curr Pharm Des 8: 3741-3753 2012.

23. Lindemann K, Christensen RD and Vergote I: First-line treatment of advanced ovarian cancer with paclitaxel/carboplatin with or without epirubicin (TEC versus TC) - a gynecologic cancer intergroup study of the NSGO, EORTC GCG and NCIC CTG. Ann Oncol 23: 2613-2619, 2012.

24. MacFarlane M, Merrison W, Bratton SB and Cohen GM: Proteasome-mediated degradation of Smac during apoptosis: XIAP promotes Smac ubiquitination in vitro. J Biol Chem 277: 36611-36616, 2002.

25. Ma L, Huang Y, Song Z, et al: Livin promotes Smac/DIABLO degradation by ubiquitin-proteasome pathway. Cell Death Differ 13: 2079-2088, 2006.

26. Fulda S, Wick W, Weller M and Debatin KM: Smac agonists sensitize for Apo2L/TRAIL- or anticancer drug-induced apoptosis and induce regression of malignant glioma in vivo. Nat Med 8: 808-815, 2002.

27. Emeagi PU, Van Lint S, Goyvaerts C, et al: Proinflammatory characteristics of SMAC/DIABLO-induced cell death in antitumor therapy. Cancer Res 72: 1342-1352, 2012.

28. Jiang G, Zhao J, Xiao X, et al: An N-terminal Smac peptide sensitizes human prostate carcinoma cells to methyl jasmonateinduced apoptosis. Cancer Lett 302: 37-46, 2011.

29. Xiao XY, Jiang GS, Wang L, Lv L and Zeng FQ: Predominant enhancement of apoptosis induced by methyl jasmonate in bladder cancer cells: therapeutic effect of the Antp-conjugated Smac peptide. Anticancer Drugs 22: 853-863, 2011. 